\title{
Perception of three-dimensional angular rotation
}

\author{
J. TIMOTHY PETERSIK \\ Ripon College, Ripon, Wisconsin
}

\begin{abstract}
In three experiments, difference thresholds (dLs) and points of subjective equality (PSEs) for three-dimensional (3-D) rotation simulations were examined. In the first experiment, observers compared pairs of simulated spheres that rotated in polar projection and that differed in their structure (points plotted in the volume vs. on the surface), axis of rotation (vertical, $y$, vs. horizontal, $x$ ), and magnitude of rotation $\left(20^{\circ}-70^{\circ}\right)$. DLs were lowest $(7 \%)$ when points were on the surface and when at least one sphere rotated around the $y$-axis and varied with changes in the independent variables. PSEs were closest to objective equality when points were on the surface of both spheres and when both spheres rotated about the $x$-axis. In the second experiment, subjects provided direct estimates of the rotations of the same spheres. Results suggested a reasonable agreement between PSEs for the indirect-scaling and direct-estimate procedures. The third experiment varied sphere diameter (and therefore mean linear velocity of stimulus elements) and showed that although rotation judgments are biased by mean linear velocity, they are not likely to be made solely on the basis of that information. These and past results suggest a model whereby recovery of structure is conducted by low-level motion-detecting mechanisms, whereas rotation (and other) judgments are based on a higher level representation.
\end{abstract}

The capacity of the human visual system to discriminate stimuli varying by only small amounts on one or more dimensions is well known. With regard to linear motion, for example, velocity difference thresholds (dLs) for sinewave gratings can be as low as $1 \%-2 \%$ or as high as $40 \%-50 \%$, depending upon their spatial frequencies and drift rates (Pantle, 1978, Experiment 3). Nonetheless, despite a growing interest in the process whereby the visual system recovers three-dimensional (3-D) structure from two-dimensional (2-D) image motion (e.g., Braunstein, 1976; Braunstein \& Tittle, 1988; Hildreth, 1984; Lappin, Doner, \& Kottas, 1980; Petersik, 1979, 1980a, 1987; Sperling, Landy, Dosher, \& Perkins, 1989; Ullman, 1979), relatively little work has been done on the basic psychophysics of the 3-D motion percept produced with rotation simulations. For example, only Kaiser $(1990,1991)$ has asked what the $\mathrm{dL}$ is for pairs of objects in 3-D rotation. The present experiments, although conceived independently, extend the issues addressed by Kaiser $(1990,1991)$, as well as her data.

Kaiser's (1990) stimuli were "pseudoshaded" simulations of solid cubes (and in one case, a bispherenoid) displayed in polar projection. In separate experiments, she examined the effects of pairs of stimuli rotating about

I am grateful to G. Andersen, M. Braunstein, P. Werkhoven, and an anonymous reviewer for their detailed and insightful comments on an earlier version of this manuscript. Diane Gardetto, Niveen Iskandar, and Ted Uczen were patient and diligent subjects in this research and are thanked heartily. Reprint requests and other correspondence should be addressed to the author at the Department of Psychology, Ripon College, P.O. Box 248, Ripon, WI 54971. parallel or orthogonal axes, rotating in the same or opposite directions, and rotating in phase compared with out of phase; she also examined the influence of the number of faces (three or four) revealed during rotation and the effect of size differences between the stimuli. In her first experiment, Kaiser found that dLs were about $10 \%$ and did not vary significantly as a function of axis orientations, similarity of rotation direction, or relative phase of the stimuli. Furthermore, points of subjective equality (PSEs) were not significantly different from objective equality. PSEs were somewhat elevated when an object displaying fewer edge transitions (the bispherenoid) was compared to a rotating cube. Finally, PSEs were higher than objective equality by $18.5 \%$ when the comparison cube's sides were half the length of the standard cube's sides (a velocity transposition effect? cf. Brown, 1931). Over all experiments, subjects' dLs averaged $12 \%$. Kaiser (1990) concluded that 'observers' judgments correlate with three aspects of the stimulus displays: the depicted angular velocity, the depicted tangential velocity of surface elements, and other temporal factors (such as the rate of boundary deformation and edge transitions)"' (p. 155). She also noted that her naive subjects' performance was comparable to that of naive observers judging stimuli in translational motion.

The central question motivating the present experiments also concerned the ability of subjects to discriminate angular velocities (or, equivalently for our stimuli, rotation magnitudes). However, the stimuli used here were simulations of spheres undergoing rotation, with each sphere's structure specified by 50 or 75 pixels randomly located either on its surface or within its volume. Whereas Kaiser's 
(1990) "solid" stimuli were chosen to permit the study of tangential velocity, edge transitions, and so forth, spheres were used in the present experiments to exclude such contributions to recovery from structure. Additionally, the present stimuli were depicted under polar projection to ensure, as much as possible, that the subjects perceived the spheres as rotating in the same direction, as well as to avoid the perceptual reversals in rotation direction that can occur with orthographic projections. As was the case with Kaiser's stimuli, we also studied the influence of rotations about parallel (either $y$ - or $x$-) or orthogonal ( $y$ - vs. $x$-) axes.

The extent to which subjects can accurately judge the rotation of a stimulus in 3-D space may also be important to understanding how the perception of structure is recovered from the motion of such stimuli. For example, Cortese and Andersen (1991) simulated the rotation of ellipsoid stimuli rotating around the vertical axis by displaying their continuously deforming silhouettes. In one experiment, accuracy of shape judgments correlated with accuracy of rotation judgments. Cortese and Andersen considered a number of possible explanations for this correlation and concluded that "the perception of rotation is primary, and that the perceived shape will depend on this recovery of rotation" (p. 326). Although the stimuli of the present experiments were specified by light points that were in projective correspondence with elements of the structure of the 3-D spheres, rather than by contour deformations, the principle that shape recovery depends upon prior recovery of rotation may apply to them as well.

A final reason for conducting the present experiments was to determine the likelihood that judgments of angular rotation are based on the 3-D percept of a rotating object rather than on 2-D information contained in the display (e.g., linear velocity of stimulus elements). By varying both the size and the location of stimulus points (plotted on the surface or within the interior) of simulated rotating spheres, conditions were developed in which spheres of the same nominal sizes have elements with different mean linear velocities or in which spheres of different nominal sizes have elements with the same mean linear velocity. Comparisons of the perceived rotation magnitude of such stimuli were then used to determine the extent to which linear velocity influenced judgments.

\section{EXPERIMENT 1 Rotation dLs and PSEs}

\section{Method}

Subjects. Four subjects participated in the experiment. One was the author, and 3 were paid research assistants. Two were male (ages 20 and 39 years) and 2 were female (ages 19 and 21 years). All subjects had normal or corrected-to-normal vision; those requiring correction wore lenses during experimental sessions. The 3 research assistants had no prior experience with the type of stimuli used here, nor were they aware of the purpose or hypotheses of the experiment.
Stimuli and Apparatus. Stimuli were created and presented by an Amiga $\mathbf{5 0 0}$ microcomputer. They were presented on a Commodore $1084 S$ RGB video monitor in a noninterleaving mode (resolution: $640 \times 400$ pixels). All stimuli were simulations of two spheres undergoing rotation. Each sphere consisted of 50 randomly positioned pixels, plotted in one of two ways: either on the outer surface of the otherwise transparent sphere or within its volume. For each type of sphere, rotation was around either the $y$-axis or the $x$-axis. Each rotation simulation consisted of 15 frames, or views of the rotation, presented in rapid succession. Each of the 15 frames was displayed for $.0833 \mathrm{sec}$ (five cycles of the basic frame duration of $1 / 60 \mathrm{sec}$ ) with an interframe interval of $0 \mathrm{msec}$. Thus, each frame was refreshed five times before the next frame in the sequence was presented, leading to a frame rate of $12 \mathrm{per} \mathrm{sec}$. All possible combinations of the two factors indicated above yielded the following kinds of displays: both spheres rotating about the $y$-axis, with pixels plotted on the interior of both, surface of both, or on the surface of one and the interior of the other; both spheres rotating about the $x$-axis, with pixels plotted on the interior of both, surface of both, or on the interior of one and the surface of the other; and one sphere rotating about the $y$-axis while the other rotates about the $x$-axis, with pixels plotted on the interior of both, surface of both, or on the interior of one and the surface of the other. In the displays for which the location of stimulus points differed for the two spheres, the standard sphere always had points on the surface, whereas the comparison sphere had them on its interior. In displays for which the axis of rotation differed for the two spheres, the standard sphere always rotated about the vertical $(y$-) axis while the comparison sphere rotated around the horizontal $(x-)$ axis.

In all displays, the two spheres were presented just to the right and the left of the center of the display screen so that, at their closest, they were 1 pixel apart. Each sphere had a radius of $4.45 \mathrm{~cm}\left(5.6^{\circ}\right.$ arc). Spheres were presented in polar projection with a perspective ratio of 10.0 (simulated viewing distance divided by sphere radius). Viewing distance was approximately $44.5 \mathrm{~cm}$, which matched the simulated projection. Luminance of each white pixel was $5.8 \mathrm{~cd} / \mathrm{m}^{2}$. Light reflected from the black background was approximately $1.1 \mathrm{~cd} / \mathrm{m}^{2}$. Brightness was adjusted to minimize visible persistence of the monitor's phosphor.

The standard sphere always rotated through $45^{\circ}$ in a display. The comparison sphere rotated through $20^{\circ}, 25^{\circ}, 30^{\circ}, 35^{\circ}, 40^{\circ}, 45^{\circ}$, $50^{\circ}, 55^{\circ}, 60^{\circ}, 65^{\circ}$, or $70^{\circ}$. Because the displays always consisted of 15 frames, the angular velocity of the comparison sphere varied. Table 1 shows the angular velocity that corresponded to each rotation magnitude. When the axes of rotation were parallel, both spheres always rotated in the same direction.

A single pair of spheres was used for each complete block of trials encompassing all conditions. New spheres were prepared between blocks of trials, and the left-right positions of standard and comparison trials alternated over blocks. Uniform density, or spread, of stimulus dots was determined by visual inspection upon the creation of stimulus spheres.

Procedure. The experimental room was illuminated by only the monitor screen and a shaded 40-W incandescent bulb placed near the experimenter. Viewing was always binocular. Sessions were divided into three groups, run in the following order: both spheres rotating around the $y$-axis, both spheres rotating around the $x$-axis,

Table 1

Angular Velocity (deg/sec) as a Function of Magnitude of Rotation (deg) Magnitude of Rotation

\begin{tabular}{lllllllllllll}
\hline \multicolumn{10}{c}{ Magnitude of Rotation (deg) } \\
\cline { 2 - 11 } & 20 & 25 & 30 & 35 & 40 & 45 & 50 & 55 & 60 & 65 & 70 \\
\hline Angular Velocity & 16 & 20 & 24 & 28 & 32 & 36 & 40 & 44 & 48 & 52 & 56 \\
\hline
\end{tabular}


and mixed rotation. Within each group, sessions were blocked so that subjects ran trials with interior/interior points, surface/surface points, and interior/surface points in a random order. Within a block, the rotation magnitude of the comparison sphere was varied randomly.

On any trial, two motionless spheres appeared on the screen to warn the subject that a trial was about to begin. Within the next $5 \mathrm{sec}$, the spheres began their rotation and continued for four cycles or until the subject responded. During a trial, the subject stared at the point just between the two spheres. The subject's task was to report which sphere, the right or the left, was rotating faster. Since angular velocity and rotation magnitude were perfectly correlated, the subjects were also allowed to base their judgments on rotation magnitude alone. ${ }^{1}$ No feedback was provided.

\section{Results}

For each subject in each condition of the experiment, a dL and a PSE were calculated by Spearman's method (Engen, 1972). ${ }^{2}$ Since there were no differences in the qualitative appearance of the 4 subjects' data, and since the resulting standard errors were quite low, data were grouped for analysis. ${ }^{3}$ The dLs and PSEs are considered individually below.

dLs. The average absolute dLs that were obtained in the experiment are shown in Figure 1, in which $\mathrm{dL}$ (in degrees) is plotted as a function of axes of rotation. Each curve shows the data from one condition of the locationof-points factor. As can be seen, dLs were lowest (about $3.25^{\circ}$, or about $7 \%$ of the standard sphere's rotation of $45^{\circ}$ ) when points were simulated to lie on the surface of both spheres and when the rotation of at least one sphere was around the $y$-axis. In general, dLs were smallest for conditions in which points were on the surfaces of both spheres, followed by conditions in which points were within the interiors of both spheres, and were largest when the points were on the surface of one sphere and on the interior of the other.

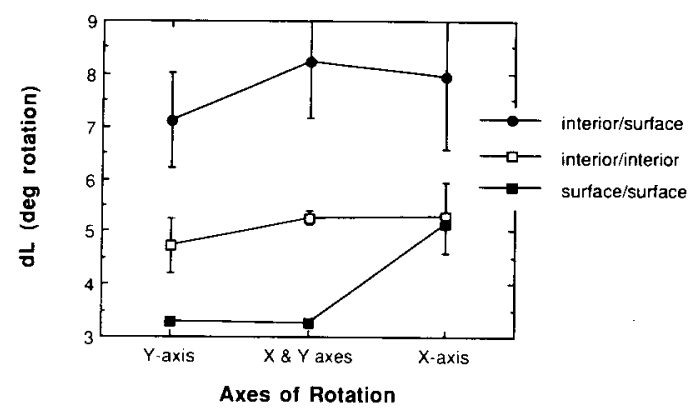

Figure 1. Mean absolute difference threshold (dL) as a function of axes of rotation: $y$-axis - both spheres rotated around the $y$-axis; $x$ and $y$-axes-one sphere rotated around the $x$-axis while the other sphere rotated around the $y$-axis; $x$-axis-both spheres rotated around the $x$-axis. Separate curves show results from different conditions of the location-of-points factor: interior/surface-one sphere had points simulated to be on the interior of the sphere, whereas the other had points simulated to be on the surface; interior/interior both spheres had points simulated to be on the interior; surface/ surface-both spheres had points simulated to be on the surface. Error bars show \pm 1 SEM.
A completely repeated measures analysis of variance (ANOVA) revealed a significant main effect of the simulated locations of points $[F(2,6)=47.03, p<.008]$ : The mean dLs when points were plotted on the surface of both spheres, when points were plotted within the interior of both spheres, and when the spheres differed with regard to where the points were plotted, were $3.89^{\circ}(8.6 \%)$, $5.08^{\circ}(11.3 \%)$, and $7.76^{\circ}(17.3 \%)$, respectively. Analytical comparisons (as described in Keppel, 1982, chap. 18) showed that there was no significant difference between the two conditions in which points were plotted in the same way for both spheres $[F(1,6)=2.19$, $p>.05]$. However, the condition in which one sphere had points plotted on the interior while the other had them plotted on the surface was significantly different from each of the other two conditions: $F(1,6)=11.12, p<.05$, when compared with the condition in which points were always plotted on the interior, and $F(1,6)=23.18$, $p<.01$, when compared with the condition in which points were always plotted on the surface. Thus, it is clear that dLs were significantly larger when the subjects compared spheres that differed in the locations of points that specified their structures-that is, points within the sphere's volume as opposed to points on the surface only. This effect could have been due to a difference in the mean linear velocities of stimulus points in the two kinds of stimuli. The contribution of linear velocity to rotation judgments is considered further in Experiment 3.

The repeated measures ANOVA failed to reveal a significant effect of the axis of rotation $[F(2,6)=3.64, p=$ $.092]$. The fact that the differences are nearly significant can probably be explained solely by the large mean $\mathrm{dL}$ in the condition in which points were always plotted on the surface and both spheres rotated around the $x$-axis (see Figure 1). This data point differs from our pilot data, which, for this condition, yielded a mean $\mathrm{dL}$ of about $4^{\circ}$.

Finally, the axis of rotation $\times$ location of points interaction failed to show significance $[F(4,12)=1.43$, $p>$.05].

PSEs. The average absolute PSEs (rotations of the comparison spheres required to be perceived as equal to the $45^{\circ}$ rotation of the standard sphere) obtained in Experiment 1 are shown in Figure 2, in which PSE is plotted as a function of axes of rotation. Each curve shows the data from one condition of the location-of-points factor. The condition yielding the PSE closest to objective equality $\left(45.06^{\circ}\right)$ was that in which points were plotted on the surface of both spheres and both spheres rotated about the $x$-axis. As can be seen, the conditions in which points were plotted in different locations within the two spheres consistently led to PSEs that were larger than objective equality $\left(45^{\circ}\right.$ ) by more than $5^{\circ}$ (more than roughly $11 \%$ ) - that is, the spheres with points plotted on the interior had to rotate faster than the standard to be seen as equivalent to it. On the other hand, the conditions in which points were always plotted in the same locations with respect to the two spheres (either on the surfaces or within 


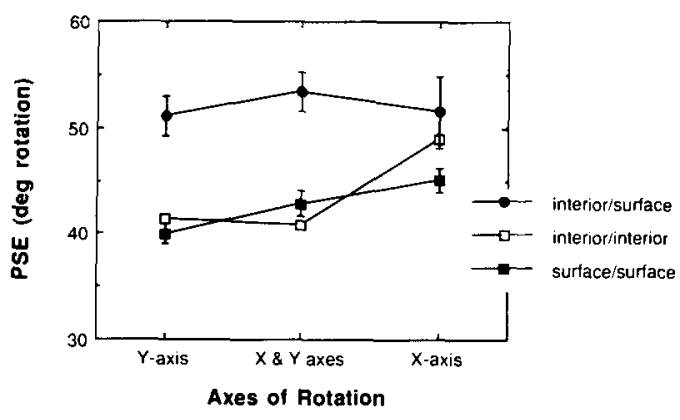

Figure 2. Mean absolute point of subjective equality (PSE) as a function of axes of rotation. Conventions as in Figure 1.

the volumes) tended to lead to PSEs that were smaller than objective equality, but by $5^{\circ}$ or less.

A repeated measures ANOVA of the PSEs showed that both factors and the interaction were significant. The significant effect of point locations $[F(2,6)=16.07, p<$ .005] was apparently due to the significantly higher mean PSE that was obtained when the points of the two spheres differed with respect to their locations $\left(52.06^{\circ}\right)$ compared with the average of the two conditions in which points were in the same locations $\left[43.14^{\circ} ; F(1,6)=30.90\right.$, $p<.011$.

The significant effect of axis of rotation $[F(2,6)=6.43$, $p<.05]$ was due to the difference between the mean PSE obtained when both spheres rotated around the $x$-axis $\left(48.48^{\circ}\right)$ and the average of the remaining two conditions $\left[44.91^{\circ} ; F(1,6)=11.25, p<.05\right]$.

An analysis of the significant interaction $[F(4,12)=$ $3.451, p<.05]$ showed that when the two spheres had disparate point locations (i.e., one with points in the volume, the other with points on the surface), PSEs were not influenced by axis of rotation $[F(2,6)=1.10, p>$ $.05]$, whereas the average PSEs in the two conditions in which the points were located similarly in the two spheres were influenced significantly by axis of rotation [see Figure $2 ; F(2,6)=10.71, p=.011]$.

On the basis of these results, it appears as though PSEs did not differ when the points in both the standard and the comparison spheres were plotted in the same locations, that these PSEs were slightly below objective equality, and that these PSEs changed significantly with changes in the axis of rotation. On the other hand, when the points were plotted differently in the standard and comparison spheres, PSEs did not change as a function of axis of rotation and were somewhat higher than objective equality. Once again, this effect could be due to the difference in the mean linear velocity of elements in the "interior" and "surface" spheres.

It is worth noting that in Experiment 1 there was little difference in either dL or PSE for conditions in which both stimuli were plotted with points on the interior as opposed to conditions in which both stimuli were plotted with points on the surface, despite the fact that the mean linear velocity of stimulus elements differed by a factor of approximately two between these conditions (with the surface spheres having the greater mean linear velocity). The similarity of dLs and PSEs for these pairs of conditions suggests that rotation judgments were made on the basis of a 3-D percept of angular rotation rather than on the basis of 2-D linear velocity.

\section{EXPERIMENT 2 Direct Estimates of Rotation Magnitude}

In Experiment 1, the subjects made judgments in accord with classical methods-that is, they provided judgments of relative magnitude in response to pairs of stimuli consisting of a standard and a comparison stimulus. To ensure that dLs obtained by the classical method were not based solely on the linear 2-D velocity of the stimulus elements (since projected 2-D velocity of stimulus elements is proportional to the 3-D angular rotation of the objects being simulated in these displays), two additional experiments were conducted. In Experiment 2, the subjects were asked to provide direct estimates of the angular extent of rotation in 3-D simulations. Evidence that estimated extents of rotation are similar to objective rotations would suggest that the relative judgments of Experiment 1 were based on the 3-D rotation percept rather than on 2-D velocity per se. Additionally, the data from Experiment 2 can be used to understand the form of the relationship between objective rotation magnitude and perceived rotation magnitude. Experiment 3 was conducted to determine the possible influence of the projected 2-D velocity of stimulus elements on perceived rotational magnitude.

\section{Method}

Subjects. The same 4 subjects who had previously served in Experiment 1 participated.

Stimuli and Apparatus. Experimental stimuli and apparatus were the same as in Experiment 1, except that only one sphere was judged on each trial.

Procedure. Stimuli were block-randomized and presented as they were in Experiment 1. Now, however, the subject's task was to rotate a circular protractor affixed to a square piece of cardboard so that the rotation of the protractor had the same magnitude and direction as the comparison stimulus in each display (the subject knew before seeing the display which stimulus was to be judged). A line on the cardboard served as a reference point for judging the extent of rotation. On each trial, the subject had unlimited time to make the judgment; however, most trials lasted less than $10 \mathrm{sec}$. After each judgment, an experimenter read and recorded the protractor's rotation. The subjects served in seven replications of each condition.

\section{Results}

Rotation around the $y$-axis. Figure 3 shows the results obtained when the target stimulus rotated around the $y$ axis. Separate panels show data obtained when stimulus points represented the volume (interior) of the sphere as opposed to its surface. Data and means from individual subjects are shown. A variety of curve-fitting procedures were attempted, and the one accounting for the greatest amount of variance (in each case, more than $99 \%$ ) is 

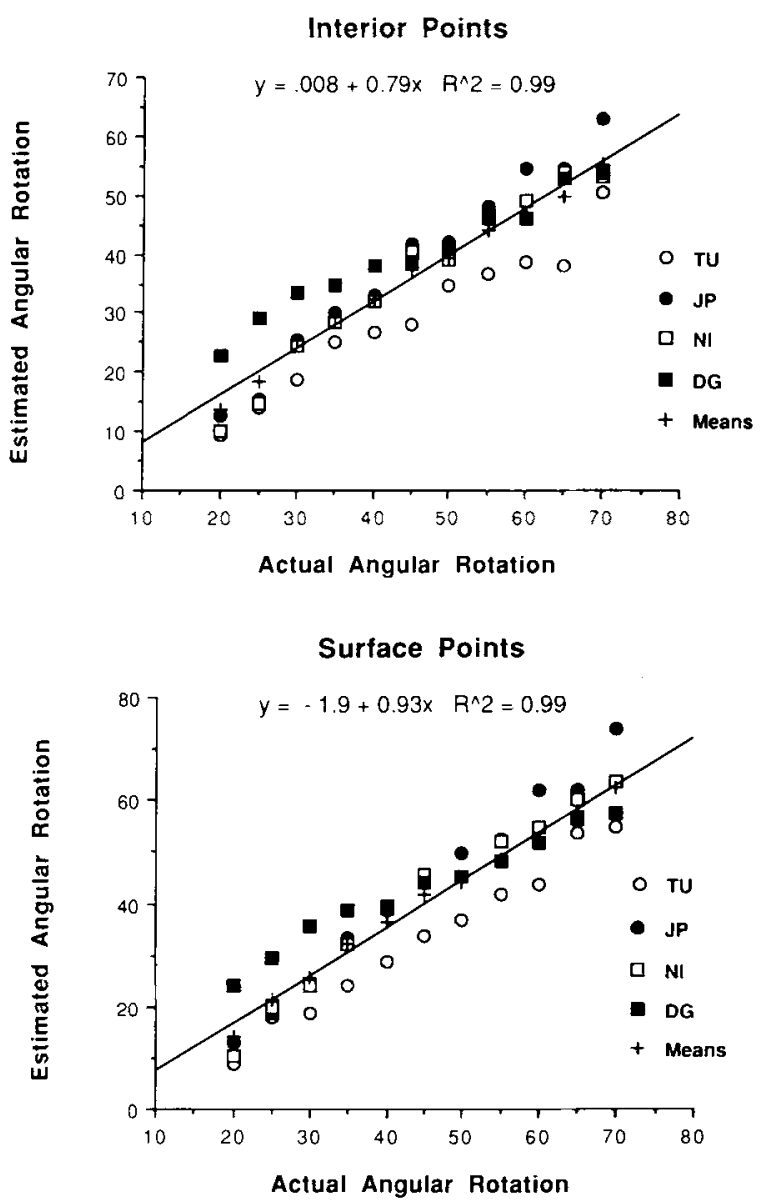

Figure 3. Estimated angular rotation as a function of actual angular rotation for conditions in which spheres rotated around the $y$-axis. Separate symbols show data and means from individual subjects. Solid lines show the best-fitting linear-regression equations. Separate graphs show data from conditions in which spheres were simulated to have their points plotted either on the interior or on the surface.

shown in each panel. For these conditions, the best-fitting lines were linear. Both lines have $y$-intercepts close to $0^{\circ}$ and slopes less than 1.0 (with the spheres represented by interior points showing the greatest departure from unity). By using the regression line, it was determined that the expected PSE when points were plotted on the interior of the sphere was $35.56^{\circ}$; the corresponding PSE obtained in Experiment 1 was $41.31^{\circ}$. It was determined from Figure 3 that the expected PSE when points were plotted on the surface of the sphere was $39.95^{\circ}$; the corresponding PSE obtained in Experiment 1 was $39.94^{\circ}$. The difference between the PSEs estimated in Experiments 1 and 2 is less than the value of $2 S D$ s in either data set.

These results show that for stimuli rotating around the $y$-axis, there is a reasonable agreement between PSEs obtained indirectly (i.e., through scaling) and those obtained directly. This further suggests, although not unequivocally, that in Experiment 1 the subjects relied on percepts of 3-D rotation to make their comparative judgments. The results also show that there is a linear relationship between objective and perceived rotation magnitude, with the percept growing somewhat more slowly than the stimulus in the case of spheres simulated by points plotted within their volume.

Rotation around the $x$-axis. Figure 4 shows the results obtained when the target stimulus rotated around the $x$ axis. Again, the data are shown for individual subjects, as well as for the group. Once again, the curves that accounted for the most variance are shown. These curves are not similar to those obtained when rotation was around the $y$-axis. First, when points were plotted on the spheres' interiors, the resulting function was not linear. Instead, the best fitting curve was logarithmic, showing that the growth in the percept slowed considerably as rotation was increased. Here, the PSE estimated from the logarithmic function was $39.89^{\circ}$; the PSE obtained for this condition in Experiment 1 was $48.88^{\circ}$. The difference between these two estimates is nearly the value of $9 S D$ s of the data from Experiment 1.
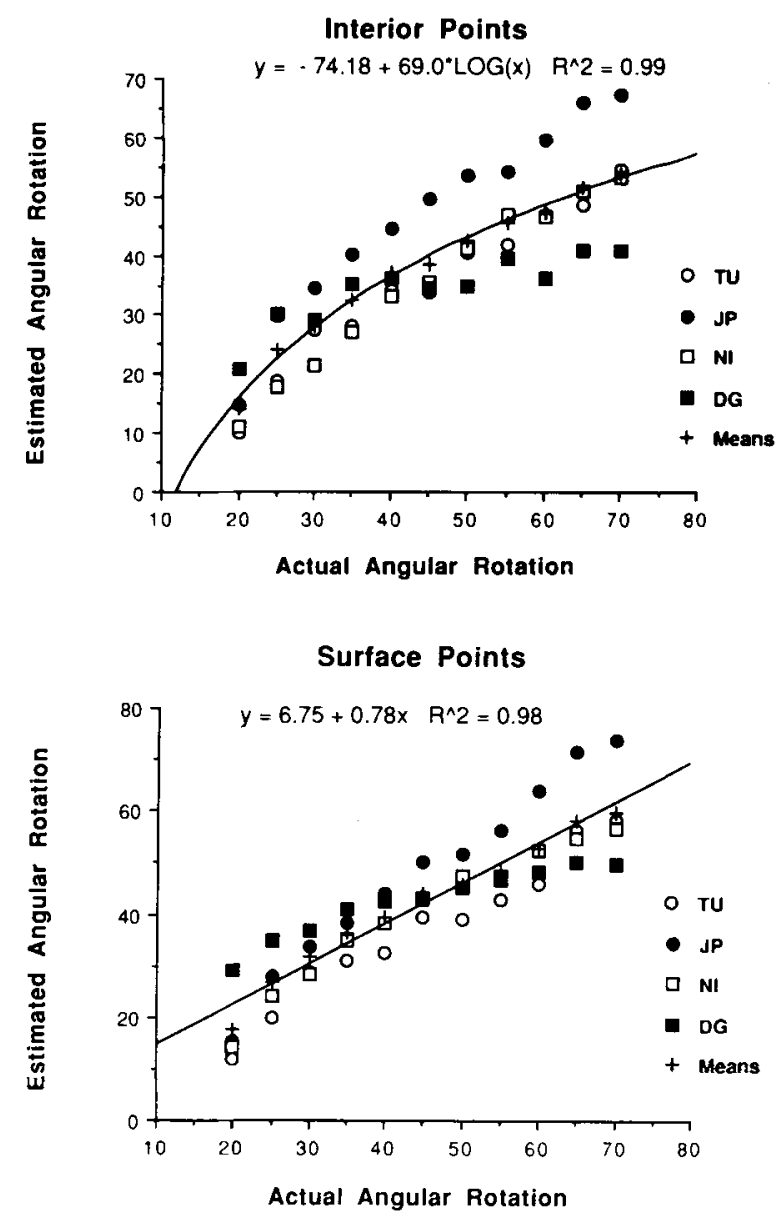

Figure 4. Estimated angular rotation as a function of actual angular rotation for conditions in which spheres rotated around the $\boldsymbol{x}$-axis. Conventlons are as in Figure 3, except that the best-fitting curve for the condition in which points were simulated to be on the interior is logarithmic, not linear. 
However, when the points were plotted on the surfaces of the spheres, the resulting data were again fit best by a linear function. In this case, the $y$-intercept was $6.75^{\circ}$ and the slope was 0.78 . The estimated PSE was $41.85^{\circ}$; the corresponding PSE obtained in Experiment 1 was $45.06^{\circ}$. The difference was again less than the value of $2 S D$ s from either data set.

The data from the direct-estimate experiments suggest two conclusions: First, the growth of the rotation percept lags considerably behind the growth in objective rotation for spheres in which points are plotted on the interior. Second, growth of the perception of rotation lags behind objective rotation more when rotation is about the $x$-axis than when it is about the $y$-axis, suggesting an anisotropy with respect to the axes of rotation. The condition yielding the greatest similarity between actual and perceived magnitude of rotation was that in which spheres rotated about the $y$-axis and were specified by surface points. ${ }^{4}$

Since, for three of the four conditions, data obtained with the direct-estimation measure yielded PSEs within $2 S D$ s of those obtained through indirect methods, the most conservative conclusion regarding the basis of comparisons made in Experiment 1 is to suspend judgmentthat is, the evidence does not warrant clear acceptance or rejection of the hypothesis that the subjects made comparisons solely on the basis of their 3-D perception of rotation. Since the projected 2-D (linear) velocities of stimulus elements vary proportionally with the radius of the rotating sphere that comprises them, a further test of whether perception of 3-D rotation is based on 2-D (linear velocity) or 3-D (angular velocity) information is to vary the size of the rotating sphere while maintaining equal angular rotations. Results suggesting that larger spheres appear to undergo greater, or faster, rotations than smaller spheres would suggest that linear velocities at least contribute to, if not determine, the judgments. Results suggesting no difference between spheres of different sizes would provide evidence for the proposition that judgments are based on the 3-D perception of angular rotation.

\section{EXPERIMENT 3 \\ Variation of Size of Simulated Spheres}

\section{Method}

Subjects. Subjects were the same 4 individuals who participated in Experiments 1 and 2.

Stimuli and Apparatus. Apparatus was the same as that in the previous experiments, with the exception that a chin rest was provided for the comfort of the subjects. Stimuli were designed the same way as those in the previous experiments, except that there were two possible sphere sizes: small spheres (radius $=4.45 \mathrm{~cm}$, or $5.6^{\circ}$ ) were the same as the spheres used in previous experiments; large spheres had a radius twice that of the small spheres $(8.9 \mathrm{~cm}$, or $\left.11.2^{\circ}\right)$. For spheres having the same absolute radius, spheres with points plotted on the interior had a mean eccentricity of points that, on the average, was half the mean eccentricity of the spheres with points plotted on the surface. Thus, the small sphere composed of surface points and the large sphere composed of interior points had the same mean eccentricity and therefore the same mean linear velocity of points. The small sphere with points plotted on the interior had the smallest mean eccentricity (half that of the small sphere composed of surface points), whereas the large sphere with points plotted on the surface had the largest (twice that of the small sphere composed of surface points). Additionally, in order to accommodate the greater screen area occupied by the large spheres, all spheres were now composed of 75 randomly positioned pixels, rather than 50. Finally, rotation magnitudes extended from $25^{\circ}$ to $65^{\circ}$ in $5^{\circ}$ steps.

There were 72 conditions to the experiment, resulting from the factorial combination of two sphere sizes (small vs. large), two locations of stimulus points (interior vs. surface), two axes of rotation ( $y$-vs. $x$-axis), and nine levels of rotation magnitude $\left(25^{\circ}, 30^{\circ}\right.$, $35^{\circ}, 40^{\circ}, 45^{\circ}, 50^{\circ}, 55^{\circ}, 60^{\circ}$, and $65^{\circ}$ ).

Procedure. The procedure was the same as that in Experiment 2, except that eight replications of each condition were conducted with each subject.

\section{Results}

The results are displayed in Figures 5 and 6. For each figure, mean estimated rotation is shown as a function of stimulus angular rotation for some combination of stimulus-

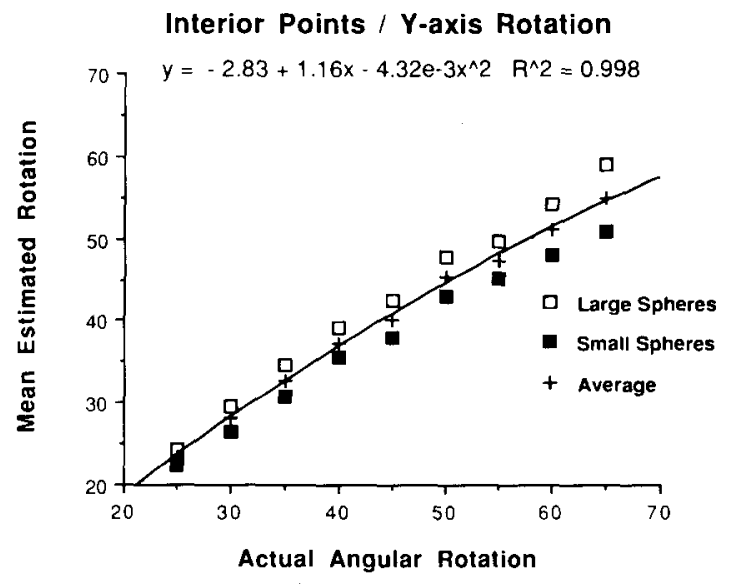

Surface Points / Y-axis Rotation

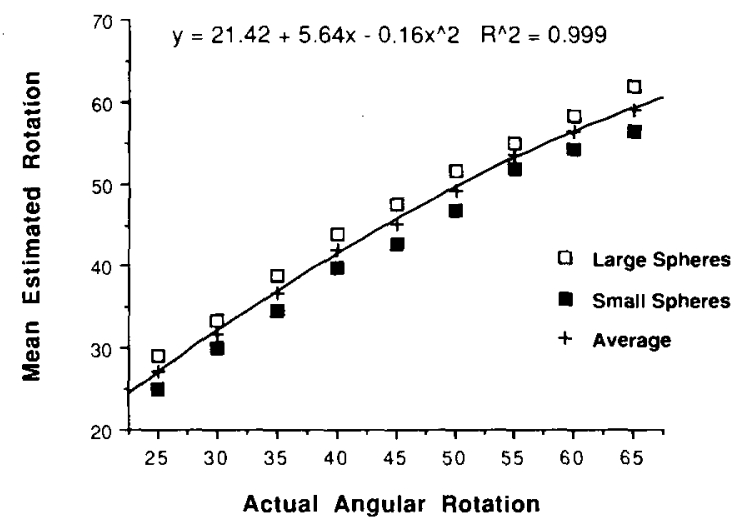

Figure 5. Mean estimated angular rotation as a function of actual angular rotation for conditions in which spheres rotated about the vertical $(y-)$ axis. Separate graphs show conditions in which stimulus elements were plotted on elther the surface or the interior of the spheres. Open symbols show data for large spheres; filled symbols show data for small spheres. The curves that beat fit the averages of the data for large and small spheres (a second-degree polynomial) are also shown, along with their equation. 
Interior Points / X-axis Rotation

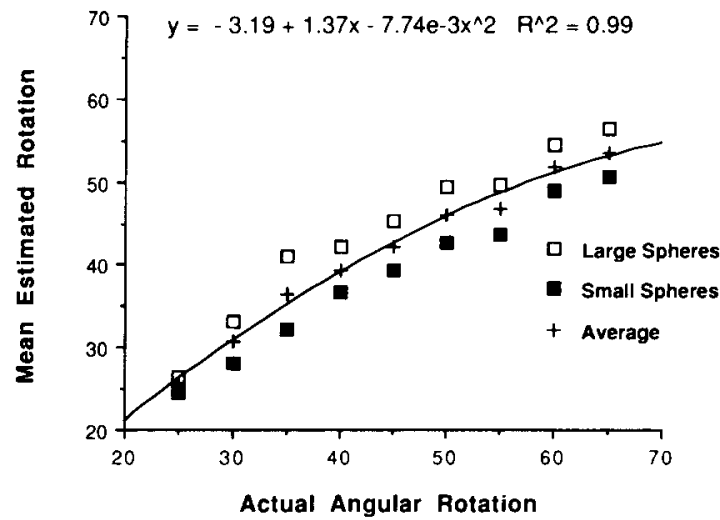

Surface Points / X-axis Rotation

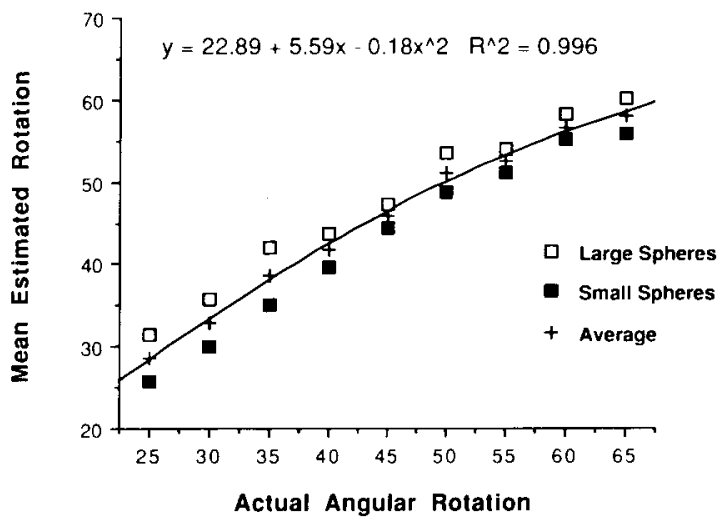

Figure 6. Same as Figure 5, except that rotation is about the horizontal $(x-)$ axis.

element location and axis of rotation. Separate sets of data are shown in each figure for large spheres versus small spheres. Also shown in each figure is the formula for the best-fitting curve (a second-degree polynomial in each case) for the average of data obtained with large and small spheres. In all four cases, the best-fitting polynomials account for only $2 \%-6 \%$ more variance than the linearregression lines. The present data confirm the trend, found in Experiment 2, that the function relating perceived rotation to actual rotation grows more slowly for spheres whose stimulus elements are plotted on the interior than for spheres whose stimulus elements are plotted on the surface.

In all cases, rotations of large spheres were perceived as having greater magnitude than the corresponding rotations of the small spheres, as shown in Figures 5 and 6 . The perceptual difference between the rotations of large and small spheres was found to be significant in a completely repeated measures ANOVA $[F(1,3)=30.15$, $p=.01]$. No significant interactions involving the size factor were obtained. Although it was significant, the influence of the size factor was not as great as might be expected if the subjects had based their judgments on linear velocities of stimulus elements, in which case the larger sphere should have received judgments twice as great as the smaller. In fact, on the average, the large spheres were fairly consistently seen as having only $5^{\circ}$ greater rotation than the small spheres. Even at the smallest absolute rotation $\left(25^{\circ}\right)$, this would make for a greater perceived magnitude of the large sphere of only $20 \%$.

In addition to the main effect of sphere size, there was a significant influence of rotation magnitude $[F(8,24)=$ $131.36, p<.001]$. However, there was no significant main effect of axis of rotation $[F(1,3)=1.21, p=.35]$. Similarly, there was no main effect of point location [surface vs. interior elements; $F(1,3)=6.31, p=.09$ ]. The ANOVA did reveal a significant point location $\times$ sphere size $\times$ rotation magnitude interaction $[F(8,24)=3.30$, $p=.011]$. Three sets of planned analytical comparisons (Keppel, 1982) were conducted to aid in the interpretation of this three-way interaction: First, a separate analysis was conducted on data from large spheres having surface versus interior elements (collapsed over axis of rotation, since that factor did not have a significant main effect in the original analysis). Because the elements of the surface spheres had a mean linear velocity that was approximately twice that of the interior spheres, a tendency on the part of the subjects to use linear velocity in arriving at judgments would lead to a significant effect of point location. However, there was no influence of point location for the large spheres $[F(1,3)=3.05$, $p=.18]$, nor was there a significant interaction between point location and rotation magnitude $[F(8,24)=1.53$, $p=.20]$. A similar analysis was also conducted on data from the small spheres. Here, there was a significant main effect of point location $[F(1,3)=15.25, p=.028]$, reflecting the fact that the small spheres comprising surface elements were perceived as rotating an average of $4.13^{\circ}$ further than the small spheres comprising interior elements. Once again, the magnitude of this difference was much smaller than would be expected if the subjects were relying solely on linear velocity to make their rotation-magnitude judgments. For the small spheres, there was no significant interaction between point location and rotation magnitude $[F(8,24)=1.06, p=.42]$. Finally, a separate analysis was conducted on data from small spheres comprising surface elements versus large spheres comprising interior elements, because these stimuli had, on the average, equivalent eccentricities and linear velocities. As was expected, the only significant factor influencing judgments of these stimuli was rotation magnitude; the stimulus type factor had no significant influence $[F(1,3)=3.69, p=.15]$ and did not contribute to a significant interaction $[F(8,24)=1.31, p=$ .29].

The lack of a significant difference between small spheres with points on the surface and large spheres with points on the interior leaves unanswered the question of the cause of the finding that perceived rotation grows more 
Table 2

Percent Deviation of Judged Angle of Rotation from Actual Angle of Rotation (deg)

\begin{tabular}{lcccccccccc}
\hline & \multicolumn{10}{c}{ Magnitude of Actual Rotation } \\
\cline { 2 - 11 } \multicolumn{1}{c}{ Stimulus } & 25 & 30 & 35 & 40 & 45 & 50 & 55 & 60 & 65 \\
\hline Large-Interior & .020 & .048 & .100 & .016 & -.024 & -.029 & -.084 & -.093 & -.109 \\
Small-Surface & .021 & .016 & .002 & -.025 & -.042 & -.069 & -.096 & -.113 & -.136 \\
\hline
\end{tabular}

Note-Positive values indicate judged rotation greater than actual; negative values indicate judged rotation smaller than actual.

slowly for spheres with points plotted on the interior than for spheres with points plotted on the surface, as well as leaving unanswered the relationship between the surface-interior difference and the overall tendency for larger spheres to be seen as having a greater rotational magnitude than smaller spheres. It is possible that both the surface-interior difference and the effect of sphere size are due to the mean eccentricity of stimulus points or linear velocity. Table 2 shows the difference between perceived and actual rotation as a percentage of actual rotation for the two conditions with equivalent eccentricities (large spheres with interior points and small spheres with surface points; both sets of data are collapsed over axis of rotation). With the exception of the findings at a rotation of $35^{\circ}$, the deviations of the two conditions are similar and follow a generally increasing trend. Although not definitive, this similarity suggests that the size and surfaceversus-interior effects may arise from a common influence, possibly linear velocity.

Collectively, the above results offer little support for the notion that the linear velocity of stimulus elements contributes in any substantial way to the judgments of rotation magnitude made in response to the present stimuli. Although both sphere size and point location had significant influences on perceived rotation in one analysis or another, neither effect approached what would be required if the subjects were making sole, or even substantial, use of linear velocity in their judgments. These results and conclusions are similar to those of Kaiser (1991).

\section{GENERAL DISCUSSION}

A number of generalizations can be gleaned from the foregoing experiments:

1. The $\mathrm{dLs}$ for the rotating-sphere stimuli used here tend to be low $(7 \%-18 \%$, with an average of about $12 \%)$ and comparable to values found with traditional, translating stimuli (as noted also by Kaiser, 1990).

2. In general, dLs were smallest for spheres with points plotted on the surface, followed by those with points plotted on the interior, and were largest when the two spheres had points in different locations.

3. Axis of rotation (vertical vs. horizontal) made no difference in dLs.

4. PSEs were generally within $5^{\circ}-8^{\circ}$ of objective equality and were larger when comparison and standard spheres differed with respect to point locations than when point locations were consistent.

5. PSEs were larger (and somewhat more accurate) when rotation was around the horizontal axis than when it was around the vertical axis, at least for comparison and standard spheres with consistent point locations.

6. Perception of the magnitude of rotation of the present stimuli is a monotonically increasing function of actual rotation, with the extent of underestimation increasing as angular rotation increases.

7. Underestimation of rotation magnitude is generally greater for spheres with points plotted on the interior than for spheres with points plotted on the surface and is also greater for spheres rotating about the horizontal axis than for those rotating about the vertical axis.

8. Although the perceived rotation associated with larger spheres is greater than that associated with smaller spheres, the difference is not large enough to warrant the conclusion that subjects base rotation judgments solely on the linear velocity of the stimulus elements.

9. The perception of the angular rotation of spheres whose stimulus elements are plotted on either the surface or the interior (and which have different mean linear velocities) is similar enough to support the suggestion that subjects base their judgments on some factor(s) in addition to linear velocity-possibly the 3-D percept itself.

10. The degree of misperception of rotation magnitude is generally an increasing function of stimulus rotation (i.e., the greater the rotation, the greater the tendency to underestimate it), with one exception-larger spheres whose elements are plotted on the surface tend to have rotations that are overestimated when stimulus rotation is relatively small $\left(25^{\circ}-40^{\circ}\right)$.

Although the present stimuli and methods were substantially different, the results of these experiments are comparable to those of Kaiser (1990). For example, her dLs averaged about $12 \%$ across experiments, whereas the average $\mathrm{dL}$ from the present Experiment 1 was $12.4 \%$ $\left(5.58^{\circ}\right)$. Similarly, Kaiser (1990) found that PSEs were elevated $18 \%$ when the standard and comparison stimuli differed structurally (i.e., in size); when the present stimuli differed with respect to the location of pixels that specified them, PSEs were elevated $15.6 \%$. Kaiser (1990) found no influence of parallel versus orthogonal axes of rotation on either dLs or PSEs; the present data also showed no substantial influence of axes on dLs, although 
the results suggested an effect on PSEs. Finally, the present results permit a conclusion that agrees with Kaiser's (1990)-namely, that the discrimination abilities of observers viewing 3-D rotation are comparable to those of observers viewing stimuli undergoing linear translation.

The subjects who viewed the present stimulus displays, as well as subjects who have viewed other displays similar to them, reported a compelling and instantaneous percept of the rotation of a 3-D object (always a sphere) about the vertical, or $y$-, axis. Furthermore, their subjective evaluation of the task was that the judgment was made, as one subject put it, by simply "watching the rotation of the globe and then imagining how far around it went." Although subjective reports rarely offer an accurate reflection of processing mechanisms, they do add to information regarding a phenomenon, and therefore models ought to be able to address subjective experiences, as well as empirical data. Three general alternatives exist regarding the nature of the processes underlying both recovery of structure from motion and judgments of rotation magnitude: (1) Information regarding rotation magnitude may develop prior to, and subsequently provide a perceptual basis for, recovery of structure from motion; (2) recovery of structure from motion may precede, and provide the perceptual basis for, judgments of rotation magnitude; and (3) both recovery of structure and perception of rotation magnitude may develop simultaneously, in parallel, and support one another. Subjective experience is consistent with the latter two alternatives (i.e., subjects believe that they base rotation judgments on the 3-D motion percept). Aspects of empirical data also support this conclusion.

Although the results of Werkhoven and Koenderink (1991) suggest that angular velocity cannot be directly recovered in 2-D rotary motion and that such judgments are based on an "average process of local linear velocity estimates" (p. 81), the present results, like those of Kaiser $(1990,1991)$, show that the single stimulus factor that accounts for most of the variance in the rotation-judgment data is, in fact, angular velocity. If some 2-D factor such as linear velocity were being used in rotation judgments, the judgments would be highly correlated with variations in that factor. However, both the present experiments and those of Kaiser $(1990,1991)$ show that linear velocity (as well as other stimulus parameters) serves mainly to "bias" judgments. Furthermore, at least one factor that would be expected to influence rotation judgments made on the basis of local computations of linear velocity, namely, element numerosity (Werkhoven \& Koenderink, 1991), has been shown not to influence rotation dLs or PSEs (Kaiser, 1991) or even the correctness of judgments of rotation direction (Petersik, 1980a). At the same time, element numerosity does influence judgments of the depth or shape of the 3-D object undergoing rotation (see, e.g., Dosher, Landy, \& Sperling, 1989). Together, the results concerning element numerosity are consistent with the notion that recovery of structure proceeds either independently of, or in parallel with, the perception of rotation magnitude.
Two other considerations also support the latter two processing alternatives identified above: First, the subjects in the present Experiments 2 and 3 were faced with the task in which they had to translate a percept of rotation into a turn of a protractor. For the subjects to perform this task accurately, it is likely that they had to operate on some mental representation of the object and perhaps conduct a mental rotation. It is difficult to believe that subjects could use, for example, linear-velocity computations to make judgments of the extent of angular rotation that seem to have required mental rotation. ${ }^{3} \mathrm{Se}$ condly, Petersik (1980b) has previously argued that rotation judgments and depth (perhaps including 3-D structure) judgments are dependent, or related. In particular, he suggested that either (1) "rotation judgments might depend on the perception of some minimum amount of depth in the rotating sphere," or (2) "the processes underlying the depth sensations and the rotation-direction sensations might have just a few mutual connections or lateral interactions" (both, p. 590).

Petersik (in press) has recently shown the likelihood that both the so-called "short-range" and "long-range" motion processes influence the 3-D percepts produced by rotation simulations. To the extent that the short-range process is low level, reflecting the activity of motiondetecting mechanisms, and the long-range process is high level, reflecting the activity of more cognitive processes, this result, combined with the arguments above, suggests the following hypothesis: The recovery of structure from motion itself is dependent on the activity of low-level motion-detecting units, whereas the judgment of various aspects of the object and its rotation is based on the mental representation itself. The two processes, motion detection and object representation, are partially dependent on one another such that stimulus manipulations that would not be mathematically required to affect the rotation percept (e.g., changes in linear velocity or axis of rotation) nonetheless can produce biases. Although this hypothesis awaits direct experimental testing, it does seem consistent with both subjective and empirical data. Furthermore, Shepard and Judd (1976) have already shown that the maximum stimulus duration permitting apparent motion in depth increases with angular rotation in a way consistent with the hypothesis of an internal 3-D representation.

\section{REFERENCES}

Braunstein, M. L. (1976). Depth perception through motion. New York: Academic Press.

Braunstein, M. L., Titrle, J. S. (1988). The observer-relative velocity field as the basis for effective motion parallax. Journal of Experimental Psychology: Human Perception \& Performance, 14, $582-590$.

Brown, J. F. (1931). The visual perception of velocity. Psychologische Forschung, 14, 249-268.

Cortese, J. M., * Andersen, G. J. (1991). Recovery of 3-D shape from deforming contours. Perception \& Psychophysics, 49, 315-327. Dosher, B. A., LANDY, M. S., SPERLuNG, G. (1989). Ratings of 
kinetic depth in multidot displays. Joumal of Experimental Psychology: Human Perception \& Performance, 15, 816-825.

ENGEN, T. (1972). Psychophysics: I. Discrimination and detection. In L. A. Riggs \& J. W. Kling (Eds.), Woodworth \& Schlosberg's experimental psychology: Vol. 1. Sensation and perception (3rd ed. pp. 11-46). New York: Holt, Rinehart \& Winston.

EPSTEIN, W. (1965). Perceptual invariance in the kinetic depth-effect. American Journal of Psychology, 78, 301-303.

HILDETH, E. C. (1984). The measurement of visual motion. Cambridge, MA: The MIT Press.

KaISER, M. K. (1990). Angular velocity discrimination. Perception \& Psychophysics, 47, 149-156.

KAISER, M. K. (1991). Factors influencing perceived angular velocity. Manuscript submitted for publication.

KePpEL, G. (1982). Design and analysis: A researcher's handbook. Englewood Cliffs, NJ: Prentice-Hall.

LAPPIN, J. S. , Doner, J. F., KotTas, B. L. (1980). Minimal conditions for the visual detection of structure and motion in three dimensions. Science, 209, 717-719.

Pantle, A. J. (1978). Temporal frequency response characteristic of motion channels measured with three different psychophysical techniques. Perception \& Psychophysics, 24, 285-294.

Petersik, J. T. (1979). Three-dimensional object constancy: Coherence of a simulated rotating sphere in noise. Perception \& Psychophysics, 25, 328-335.

Perersik, J. T. (1980a). The effects of spatial and temporal parameters on the perception of stroboscopic rotation simulations. Perception, 9, 271-283.

Petersik, J. T. (1980b). Rotation judgments and depth judgments: Separate or dependent processes? Perception \& Psychophysics, 27, 588-590.

Petersik, J. T. (1987). Recovery of structure from motion: Implications for a performance theory based on the structure-from-motion theorem. Perception \& Psychophysics, 42, 355-364.

Petersik, J. T. (in press). Effects of adaptation to apparent movement on recovery or structure from motion. Spatial Vision.

SHEPARD, R. N., JUDD, S. A. (1976). Perceptual illusion of rotation of three-dimensional objects. Science, 191, 952-954.
Speringg, G., Landy, M. S., Dosher, B. A., Perkins, M. E. (1989). Kinetic depth effect and identification of shape. Joumal of Experimental Psychology: Human Perception \& Performance, 15, 826-840.

Ullman, S. (1979). The interpretation of visual motion. Cambridge, MA: The MIT Press.

Werkhoven, P., Koenderink, J. J. (1991). Visual processing of rotary motion. Perception \& Psychophysics, 49, 73-82.

\section{NOTES}

1. It became clear after the first few sessions that no subject ever used this criterion. The subjects were questioned throughout the experiment regarding their basis of judgment, and only 1 subject reported occasionally using the magnitude-of-rotation criterion. At the same time, all subjects acknowledged an awareness that angular velocity and rotation extent were correlated in these stimuli.

2. According to Engen (1972), Spearman's method is problematical because one often must make assumptions regarding the frequencies of observations that could be obtained beyond the upper and lower limits of the comparison stimuli that were used. However, in our study, the largest and smallest rotations of the comparison stimuli always yielded frequencies of $100 \%$ and $0 \%$, respectively. Hence, no assumptions need to be made about our distributions of scores.

3. Graphs of the original psychophysical functions from each subject are available from the author on request.

4.By using a shadowcaster and a wire parallelogram, Epstein (1965) obtained rotation-magnitude estimates for physical rotations about the $y$-axis of $15^{\circ}, 25^{\circ}, 45^{\circ}, 65^{\circ}$, and $85^{\circ}$. With the use of his data, I determined the best-fitting linear equation to be $y=10.58+7.77 x$; this accounts for $99 \%$ of the variance in his data. The equation found from data obtained with this complex object is quite similar to that obtained with points plotted on the surface and rotation about the $x$-axis in the present experiment. The slope is also comparable to that presently found with points plotted on the interior and rotation about the $y$-axis.

5. I thank G. Andersen for pointing this out.

(Manuscript received August 2, 1990; revision accepted for publication July 15,1991 .) 